\title{
ARTICLE OPEN \\ Community quorum sensing signalling and quenching: microbial granular biofilm assembly
}

\author{
Chuan Hao Tan ${ }^{1,2,3}$, Kai Shyang Koh ${ }^{1}$, Chao Xie ${ }^{1}$, Joela Zhang ${ }^{3}$, Xiao Hui $\operatorname{Tan}^{4}$, Guo Ping Lee ${ }^{1,4}$, Yan Zhou ${ }^{2,3}$, Wun Jern Ng ${ }^{2,3}$, \\ Scott A Rice ${ }^{1,4,5}$ and Staffan Kjelleberg ${ }^{1,4,5}$
}

BACKGROUND: Recent reports exploring the role of gradients of quorum sensing (QS) signals in functional activated sludge have raised the question of whether shared systems of signalling synthesis and degradation, or quorum quenching (QQ), across the community inform of the means by which QS biology regulate floccular and granular biofilm assembly.

AIMS: In this study, we aimed to explore the species origin and interactive role of QS and QQ activities in such highly diverse microbial biofilm communities.

METHODS: Here, such aims were addressed systematically by a comprehensive multi-pronged RNA-sequencing, microbiological and analytical chemistry experimental approach, using two related but independently evolved floccular and granular sludge communities.

RESULTS: Our data revealed a distinct difference between the QS and QQ potentials of the two communities, with different species largely displaying either QS or QQ functions. The floccular sludge community showed a high rate of QQ activity, and this rate was dependent on the acyl chain length demonstrating specificity of degradation. When the floccular biomass was transformed into the granular sludge, the QQ activity of the community was reduced by $30 \%$. $N$-acyl homoserine lactones with four to eight carbons on the acyl chain accumulated at the granular stage, and their concentrations were at least threefold higher than those of the floccular stage. These findings corroborated meta-community analysis where a major shift in the dominant species from potential signal quenchers to producers was observed during the transition from flocs to granules, indicating the role of species composition and associated signalling activities in coordinating community behaviours.

CONCLUSIONS: This study suggests that QQ has an important function in regulating community level QS signalling, and provides a mechanistic insight into the role of QS biology in complex community assembly.

npj Biofilms and Microbiomes (2015) 1, 15006; doi:10.1038/npjbiofilms.2015.6; published online 27 May 2015

\section{INTRODUCTION}

In most natural and engineered ecosystems, bacteria predominantly reside in structured communities, in aggregations commonly known as microbial biofilms. ${ }^{1}$ These microbial consortia can be comprised of hundreds to thousands of bacterial species with varying metabolic capabilities and collectively displaying community level properties that are distinct from the planktonic cells. ${ }^{2,3}$ The overall composition and function of complex biofilms is typically driven by interactions between the different microbial species. ${ }^{1,3}$ Understanding the dynamics of interspecies interactions within such complex multi-species biofilms is challenging, yet important in order to control and regulate key ecosystem functions. These include, e.g., the engineering of highly stable and sustainable microbial communities for water and wastewater treatment, or the treatment of biofilm-related diseases.

In many cases, interspecies interactions involve communication via small diffusible signalling molecules, a mechanism generally termed quorum sensing $(\mathrm{QS})^{3}{ }^{3}$ Many bacteria use $\mathrm{QS}$ to synchronise population behaviours, including, but not limited to, biofilm formation, exoenzyme production and virulence factor secretion, to optimise population growth and survival in different environments. ${ }^{4,5}$ The $\mathrm{N}$-acyl homoserine lactone (AHL)-mediated QS system is one of the most well-characterised bacterial communication systems and is present in $~ 10 \%$ of Proteobacteria isolated from various ecological niches. ${ }^{6-8}$ As a consequence of multiple community members sharing the same classes of signalling molecules, crosstalk or communication among bacteria of different species or even between organisms from different domains is possible. ${ }^{9-12}$ For example, Burkholderia cepacia can perceive the AHLs released by Pseudomonas aeruginosa in coculture, leading to the formation of mixed microcolonies, in contrast to the formation of separate, non-mixed microcolonies in the absence of QS activity. ${ }^{10}$ AHLs are also present at biologically relevant concentrations in complex communities in a diverse range of habitats, including sputum from cystic fibrosis patients, ${ }^{13}$ microbial mats, ${ }^{14}$ the rumen ${ }^{15}$ and wastewater treatment plants. ${ }^{16,17}$ These findings suggest that community level QS signalling is likely to be important across most habitats. Indeed, the role of AHL-mediated QS in the formation of complex, mixedspecies sludge biofilms as well as the assembly of microbial granules was recently demonstrated in a membrane bioreactor ${ }^{18}$ and a sequencing batch reactor (SBR), ${ }^{19}$ respectively. For the SBR, it was found that the in situ production of specific AHLs was

\footnotetext{
${ }^{1}$ Singapore Centre on Environmental Life Sciences Engineering (SCELSE), Nanyang Technological University, Singapore; ${ }^{2}$ Advanced Environmental Biotechnology Centre (AEBC), Nanyang Environment and Water Research Institute (NEWRI), Nanyang Technological University, Singapore; ${ }^{3}$ The School of Civil and Environmental Engineering, Nanyang Technological University, Singapore; ${ }^{4}$ The School of Biological Sciences, Nanyang Technological University, Singapore and ${ }^{5}$ Centre for Marine Bio-Innovation and School of Biotechnology and Biomolecular Sciences, University of New South Wales, Sydney, Australia.

Correspondence: S Kjelleberg (LASKJELLEBERG@ntu.edu.sg)
}

Received 3 January 2015; revised 24 March 2015; accepted 7 April 2015 
strongly and positively correlated with the morphogenesis of microbial granules. Elevated concentrations of AHLs were linked to the formation of granules, whereas decreased concentrations coincided with the disintegration of granules and conversion to the flocs. ${ }^{19}$ Most importantly, these processes were closely related to the abundance of a diverse range of microbial species in the granular communities, suggesting a highly complex, AHL-based community interaction. ${ }^{19}$ However, it is unclear how such interactions can be achieved and coordinated among the individual, phylogenetically different, species in complex communities.

One possible mechanism for the regulation of QS behaviour in the environment is via the control of signal concentrations, which is critical for effective QS signalling. It is possible that signal concentration in the environment could be regulated via specific enzymatic signal degradation activity or quorum quenching (QQ) by members of the community. Although $\mathrm{QQ}$ has been demonstrated in diverse microbial species, ${ }^{20-23}$ the ecological role and the impact of $\mathrm{QQ}$ on $\mathrm{QS}$ signalling remain largely unknown. Here we have explored the role of $\mathrm{QQ}$ as a regulator or modulator of community QS signalling. Using a complex floccular sludge commnity as a model, AHL-dependent QQ was found to be active and specific, mediated by community members of diverse origins. The assembly of floccular biomass into the granular sludge biofilms was inversely correlated with the competing QS and QQ activities of the community, suggesting an important function of QQ in coordinating community level QS signalling and behaviour.

\section{MATERIALS AND METHODS}

\section{Bioreactor operation}

An SBR $(10 \times 76.4 \mathrm{~cm}$, diameter $\times$ height $)$, seeded with floccular sludge community from a water reclamation plant (Ulu Pandan, Singapore), was operated at $22{ }^{\circ} \mathrm{C}$ for 82 weeks. ${ }^{24}$ The sludge culture was fed with synthetic wastewater (SWW) comprising $150 \mathrm{mg}$ chemical oxygen demand per litre acetate and $50 \mathrm{mg}$ chemical oxygen demand per litre propionate as carbon source, $20 \mathrm{mg} / \mathrm{I}$ ammonium, $10 \mathrm{mg} / \mathrm{l}$ orthophosphate and trace nutrient elements. ${ }^{25}$ The bioreactor had a final working volume of $4 \mathrm{I}$. The bioreactor operation involved a 5-h cycle with two continuous stages of anaerobic, aerobic and anoxic periods. A total volume of 1 litre SWW was fed per cycle and resulted in a hydraulic retention time of $20 \mathrm{~h}$. Bioreactor $\mathrm{pH}$ was maintained between 6.7 and 8.2 under the control of a programmable logic controller via dosing of either $9.12 \mathrm{~g} / \mathrm{l} \mathrm{HCl}$ or $10.0 \mathrm{~g} / \mathrm{l} \mathrm{NaOH}$. System performance of the bioreactor was monitored via weekly cycle studies. Concentrations of ammonium, nitrite, nitrate and orthophosphate, as well as concentrations of sludge biomass, i.e., mixed liquor-suspended solids and mixed liquor volatile-suspended solids were determined in accordance to the American Public Health Association (APHA) standard engineering methods. ${ }^{26}$ One millilitre aliquots of sludge samples were collected at the end of the anoxic period. The sludge biomass was immediately harvested after centrifugation $(5 \mathrm{~min}, 8000 \mathrm{~g}$, $4{ }^{\circ} \mathrm{C}$ ) and stored at $-80^{\circ} \mathrm{C}$ for subsequent RNA analysis. Similarly, $50 \mathrm{ml}$ aliquots of treated effluents were kept at $-80^{\circ} \mathrm{C}$ immediately after sampling for later AHL analysis. At weeks $40-44$, a portion of the floccular sludge culture from the SBR was collected and used to seed a second SBR $(6 \times 200 \mathrm{~cm}$, diameter $\times$ height). The second SBR was operated according to the first SBR, with some modifications, to facilitate the formation of microbial granules (as previously described in detail). ${ }^{19}$ Briefly, the second SBR was operated at an hydraulic retention time of $12 \mathrm{~h}$ and the settling time for each cycle was reduced from 60 to $5 \mathrm{~min}$ within the first 5 weeks of reactor operation. ${ }^{19}$ Here granules were defined as compact aggregates with a minimum particle diameter of $100 \mu \mathrm{m}$ and a sludge volumetric index $\left(\mathrm{SVI}_{5}\right.$, a measure of biomass density/compactness) of $50 \mathrm{ml} / \mathrm{g}$ or less, ${ }^{19,27}$ while flocs were loosely aggregated biomass with particle diameter of $100 \mu \mathrm{m}$ or less and a $\mathrm{SVI}_{5}$ of at least $50 \mathrm{ml} / \mathrm{g}$.

\section{Bacterial strains and growth conditions}

AHL biosensors including Agrobacterium tumefaciens A136, ${ }^{28}$ Chromobacterium violaceum CV026 (ref. 29) and Escherichia coli pJBA357, ${ }^{30}$ as well as $P$. aeruginosa MH602 (ref. 31) and E. coli JM109 (ref. 32) were routinely maintained in Luria-Bertani medium. The following antibiotics were supplemented into the growth medium whenever necessary: tetracycline $(4.5 \mu \mathrm{g} / \mathrm{ml})$, spectinomycin $(50 \mu \mathrm{g} / \mathrm{ml})$, kanamycin $(50 \mu \mathrm{g} / \mathrm{ml})$, ampicillin $(100 \mu \mathrm{g} / \mathrm{ml})$ or gentamycin $(40 \mu \mathrm{g} / \mathrm{ml})$. The genotypes of these bacterial strains are listed in Supplementary Table S1. Cultures were incubated at $30^{\circ} \mathrm{C}$ for $24-48 \mathrm{~h}$.

\section{$\mathrm{N}$-Acyl homoserine lactones}

Synthetic AHLs (>97\%) were purchased from Sigma-Aldrich (Singapore). Each AHL was expressed as an acronym: C4-HSL ( $N$-butyryl-DL-homoserine lactone), C6-HSL ( $\mathrm{N}$-hexanoyl-DL-homoserine lactone), 30C6-HSL ( $\mathrm{N}$-(3oxohexanoyl)-DL-homoserine lactone), C7-HSL (N-heptanoyl-DL-homoserine lactone), C8-HSL (N-octanoyl-DL-homoserine lactone), 3OC8-HSL ( N-(3oxooctanoyl)-I-homoserine lactone), C10-HSL (N-decanoyl-DL-homoserine lactone), 30C10-HSL (N-(3-oxodecanoyl)-L-homoserine lactone), C12-HSL (N-dodecanoyl-DL-homoserine lactone), 30C12-HSL (N-(3-oxododecanoyl)L-homoserine lactone), 3OHC12-HSL (N-(3-hydroxydodecanoyl)-DLhomoserine lactone), C14-HSL ( $\mathrm{N}$-tetradecanoyl-DL-homoserine lactone) and 30C14-HSL ( $\mathrm{N}$-(3-oxotetradecanoyl)-L-homoserine lactone).

\section{Determination of $\mathrm{AHL}$ inactivation kinetics}

Synthetic AHLs dissolved in dimethyl sulphoxide (Sigma-Aldrich) were added individually or in combination with the sludge culture microcosms (harvested from the bioreactor) at $5 \mu \mathrm{M}$ for each $\mathrm{AHL}$, and incubated at $22{ }^{\circ} \mathrm{C}$ with constant shaking ( 200 r.p.m.). Samples were collected from each microcosm at different time points, and the sludge biomass was removed by centrifugation ( $10 \mathrm{~min}, 8000 \mathrm{~g}$ ). Residual AHLs in the sludge supernatant were extracted and quantified by high-performance liquid chromatography-tandem mass spectrometer (Shimadzu, Singapore) as described below. Heat-inactivated sludge and SWW controls were included. The adsorption of AHLs to the sludge biomass was determined by comparing the residual AHLs in the SWW and the heat-inactivated sludge controls, immediately after addition, as well as after $1 \mathrm{~h}$ incubation. The $\mathrm{pH}$ of each sludge microcosm (bulk liquid) was monitored throughout the study and ranged between 6.7 and 6.9. The impact of $\mathrm{pH}$ on the integrity of AHLs was also assessed by adding synthetic AHLs to SWW buffered at different $\mathrm{pH}$ values ranging from 6.7 to 8.3. Linear and nonlinear regression curves were modelled using Prism (GraphPad, San Diego, CA, USA ). Signal half-life was estimated based on zero and first-order kinetics. ${ }^{33}$

Detection and quantification of AHLs by high-performance liquid chromatography-tandem mass spectrometry (LC-MS/MS)

Detection and quantification of AHLs from bioreactor-treated effluents or sludge supernatants were conducted as described by Tan et al..$^{19}$ Briefly, AHLs were extracted and concentrated from the supernatants using dichloromethane (Sigma-Aldrich). The dichloromethane extracts were analysed and quantified using high-performance liquid chromatographytandem mass spectrometer system (Shimadzu LCMS8030, Shimadzu). All samples were chromatographed by high-performance liquid chromatography (Shim-pack XR-ODS C18 column) at a flow rate of $0.3 \mathrm{ml} / \mathrm{min}$. The mobile phase consisted of a linear gradient $(40-95 \%)$ of solvent B (methanol with $0.1 \%$ formic acid) and solvent $A$ ( $25 \mathrm{mM}$ ammonium formate with $0.1 \%$ formic acid). Effluents were ionised by electrospray ionisation under positive mode and detected using the multiple reaction monitoring approach. ${ }^{14,34}$ Matrix-matched multiple reaction monitoring experiments were conducted for all AHLs. ${ }^{19}$ Each standard multiple reaction monitoring profile, including specific liquid chromatography (LC) retention time, appearance of precursor ion $\mathrm{m} / \mathrm{z}$ and two transition ions, as well as relative intensity of the two transition ions, was used as a reference. To confirm the identity of the putative AHLs, a full scan ranging from $\mathrm{m} / \mathrm{z}$ 100 to 350, coupled with precursor ion scan mode, was conducted in comparison with the standard AHLs. ${ }^{14}$ For AHL quantification, matrixmatched standard curves, ranging from 0.5 to $200 \mu \mathrm{g} / \mathrm{l}$, were constructed. Two transition ions, characteristic of the respective AHLs, were used to identify the $\mathrm{AHL}$, and the transition ion with highest intensity was used to construct the standard curves. The analyte peak areas were integrated using LabSolutions (Shimadzu). The limits of detection and quantification for each AHL were calculated with a signal-to-noise ratio of 3.3 and 10 , respectively. ${ }^{14}$ The amount of each putative $\mathrm{AHL}$ in the sample was calculated based on the standard curve and the extraction efficiency of the respective AHL. The extraction efficiency for each AHL was calculated based on the recovery of the standard AHLs added at 5 and $50 \mu \mathrm{g} / \mathrm{l}$ to the heat-inactivated sludge supernatant sample matrix. Blank injections were performed at the intervals of sample injections to avoid sample carryover. 
Isolation of sludge community members

A total of five independent isolation experiments were performed from weeks 40 to 44 of the bioreactor operation. Each time, $20 \mathrm{ml}$ of uniformly mixed sludge samples were collected from the bioreactor at the end of an operational cycle. Sludge samples were vortexed vigorously for $5 \mathrm{~min}$ to disperse the floccular cells, serially diluted and spread onto the following culture media: nutrient broth, R2A, Pseudomonas isolation, SWW and $\mathrm{ABT}^{35}$ supplemented with $1.5 \%(\mathrm{w} / \mathrm{v})$ agar each. The plates were incubated at $22^{\circ} \mathrm{C}$ for 5 days. A total of 330 strains with distinct colony morphologies were isolated and identified by ribosomal DNA (rDNA) gene sequencing.

\section{Identification of isolates}

The universal primers 27F (5'-AGAGTTTGATCMTGGCTCAG-3') and 1492R (5'-ACGGTTACCTTGTTACGACTT-3') targeting the 16S rDNA gene were used to sequence bacteria, ${ }^{36}$ while primers ITS1 (5'-TCCGTAGGTGAAC CTGCGG-3') and ITS4 (5'-TCCTCCGCTTATTGATATGC- $\left.3^{\prime}\right)$ were used to sequence the internal transcribed spacer region to identify fungi. ${ }^{37}$ Sequencing was performed using the Big Dye Terminator (Applied Biosystems, Singapore). The sequences were assembled using DNA Baser sequence assembler v3.5.2 (Heracle Biosoft, www.DnaBaser.com) and matched using online sequence similarity search tools, such as BLAST, Greengenes and SILVA. The isolate sequences obtained in this study are available via GenBank under accession numbers KC252636-KC252965.

\section{Screening of $\mathrm{AHL}$ producers}

For the A. tumefaciens A136 bioassay, ABT indicator agar ${ }^{35}$ with $50 \mu \mathrm{g} / \mathrm{ml}$ of 5-bromo-4-chloro-3-indolyl-b-D-galactopyranoside (X-gal) was prepared while Luria-Bertani agar was used for the C. violaceum CV026 bioassay. Ten microlitres of overnight cultures of biosensors were spotted onto the indicator plates, while $10 \mu \mathrm{l}$ overnight cultures of isolates were placed $5 \mathrm{~mm}$ apart from the biosensor spot and incubated at $30^{\circ} \mathrm{C}$ for $48 \mathrm{~h}$. AHLs produced in situ were detected using E. coli JBA357 biosensor. Briefly, $100 \mu \mathrm{l}$ of $5 \times$ diluted $E$. coli JBA357 overnight culture were added to $100 \mu \mathrm{l}$ of samples in a 96-well plate and incubated at $22^{\circ} \mathrm{C}$ with constant shaking at 200 r.p.m. for $4 \mathrm{~h}$ before examination by confocal laser scanning microscope (Zeiss LSM 710, Carl Zeiss Pte. Ltd., Singapore) at an excitation/ emission wavelength of 488/522-535 nm. E. coli JM109, P. aeruginosa MH602 and synthetic AHLs were included as controls.

\section{Screening of $\mathrm{AHL}$ quenchers}

AHLs (30C6-HSL, 30C8-HSL and 30C12-HSL) were added individually to the overnight cultures of isolates at $5 \mu \mathrm{M}$, and incubated at $22^{\circ} \mathrm{C}$ with constant shaking at 200 r.p.m. for $2 \mathrm{~h}$. After centrifugation and ultraviolet sterilisation, residual AHLs were quantified using the A. tumefaciens A136 bioassay. ${ }^{38,39}$ Briefly, six agar bars with a width of $1 \mathrm{~cm}$ each were aseptically carved from the ABT indicator plates. Five microlitres of samples were spotted at one end of the agar bars. Approximately $0.5 \mu \mathrm{l}$ of overnight culture of the $A$. tumefaciens A 136 was spotted at progressively further distances from the loaded samples. The plates were incubated at $30{ }^{\circ} \mathrm{C}$ for $24 \mathrm{~h}$. AHLs added to Luria-Bertani at pH 6.7, 7.2 and 10.2, and E. coli JM109 cultures were included as controls. The $\mathrm{pH}$ for all overnight cultures were determined to be $<7.3$ by the end of the experiments.

\section{Phylogenetic analysis}

The 16S rDNA sequences were aligned by ClustalW Multiple alignment package (http://www.mbio.ncsu.edu/bioedit/bioedit.html) and a consensus region covering all the sequences was selected for further analysis. The aligned sequences were subjected to phylogenetic tree construction using the neighbour-joining method ${ }^{40}$ provided by the MEGA4 (http://www. megasoftware.net/mega4/mega.html). Maximum likelihood bootstrap analyses were carried out with 1,000 replicates.

\section{RNA-sequencing analysis}

Total RNA was extracted from sludge using FastRNA Pro Soil-Direct kit (MP Biomedicals, Singapore) according to the manufacturer's guidelines. Extracted RNA was subjected to DNA removal using the TURBO DNAfree kit (Applied Biosystems). Total RNA, $200 \mathrm{ng}$, was used for complimentary DNA library preparation according to the manufacturer's instructions (Illumina, Singapore). Each complimentary DNA library was ligated with a unique adaptor sequence for sample multiplexing. A total of 16 different complimentary DNA libraries were pooled and sequenced by MiSeq
System (Illumina). The RNA-sequencing data were analysed using a fast tag-based method as described. ${ }^{19}$ Briefly, a universal primer (5'-CGA CRRCCATGCANCACCT-3') for the 16S rRNA hypervariable region (V6) was used to scan each sequencing read to obtain all 33 nucleotide tag sequences downstream of the primer, and the 33 nucleotide sequences are defined as the $\mathrm{V} 6 \mathrm{tag}$ of the 16S rRNA gene. The universal primer matches $94 \%$ of $16 \mathrm{~S}$ rRNA sequences in the RDP database. ${ }^{41}$ To eliminate sequencing error-derived $\mathrm{V} 6$ tags, only $\mathrm{V} 6$ tags that were observed in at least two different reads were kept for analysis. In addition, V6 tags were removed when any single type of sequencing read accounted for $\geqslant 50 \%$ of all reads covering that V6 tag. This was done to eliminate sequencing artefacts where identical duplicated reads were generated from single templates.

\section{Statistical analysis}

All statistical analyses were conducted using Prism (GraphPad) or R (www. r-project.org). Holm-Sidak's multiple comparison tests were performed to compare the half-lives of AHLs in different samples or to examine AHL expression between different sludge samples. Pearson correlation coefficients were determined, and false discovery rate corrections were made for all multiple correlations. The correlation matrixes were clustered using unsupervised hierarchical clustering method based on Euclidean distance with complete linkage as coded by Cluster 3.0 and visualised using Java TreeView (Open Source Clustering Software, Tokyo, Japan).

\section{RESULTS}

The floccular sludge community degrades AHLs differentially based on acyl chain length

A highly diverse floccular sludge community bioreactor undergoing simultaneous nitrification, denitrification and phosphorus removal was used as the experimental system (Supplementary Figure S1). To determine whether AHL-specific QQ activity was present in this model system, synthetic AHLs were added individually or as a mixture to the floccular sludge to characterise the rates of signal degradation. Approximately $10-30 \%$ of the AHLs were lost due to adsorption to the biomass, while loss of the remaining $70-90 \%$ of signal was attributed to biological activity (Supplementary Figure S2). This was evident by the substantially shorter AHL half-lives in the presence of live sludge than when incubated in the presence of heat-inactivated sludge $(P<0.05$ for all AHLs; Figure 1). In the presence of live sludge, the signal halflife was inversely proportional to the length of the acyl chain, regardless of the substitution at the $\mathrm{C} 3$ position (Figure 1). For example, the half-lives for short-chain signals C6-HSL and 30C6HSL were $1.95 \pm 0.45$ and $2.57 \pm 0.29 \mathrm{~h}$, respectively, whereas the half-lives of the long-chain signals $\mathrm{C} 12-\mathrm{HSL}$ and $30 \mathrm{C} 12-\mathrm{HSL}$ were considerably shorter at $0.79 \pm 0.08$ and $0.66 \pm 0.06 \mathrm{~h}$, respectively. The $\mathrm{pH}$ of the live sludge microcosm was consistent at 6.7-6.9, and the data for the SWW-buffered controls indicated that there was little spontaneous degradation of AHLs between $\mathrm{pH}$ values of 6.7-8.3 (Supplementary Figure S3). Therefore, it is likely that the loss of AHLs observed here was due to active enzymatic digestion rather than passive, chemical degradation.

\section{A significant proportion of the floccular sludge community} members either produce or quench AHLs

To gain a further insight into the relationships between the specificity of $\mathrm{QQ}$ activity and signal production in the floccular sludge community, 307 strains of bacteria and 23 strains of fungi, corresponding to 50 bacterial and four fungal genera were isolated and tested for their capability to produce or degrade AHLs (Supplementary Table S2). The bacterial isolates represented $3.5 \%$ of the total community members identified by RNAsequencing (Supplementary Tables S3 and 4). AHL production was primarily assessed based on activation of different biosensors, including $E$. coli JBA357, ${ }^{30} A$. tumefaciens $\mathrm{A} 136^{28}$ and C. violaceum $\mathrm{CV}_{026}{ }^{29}$ The $\mathrm{AHL}$ profile of each putative signal 

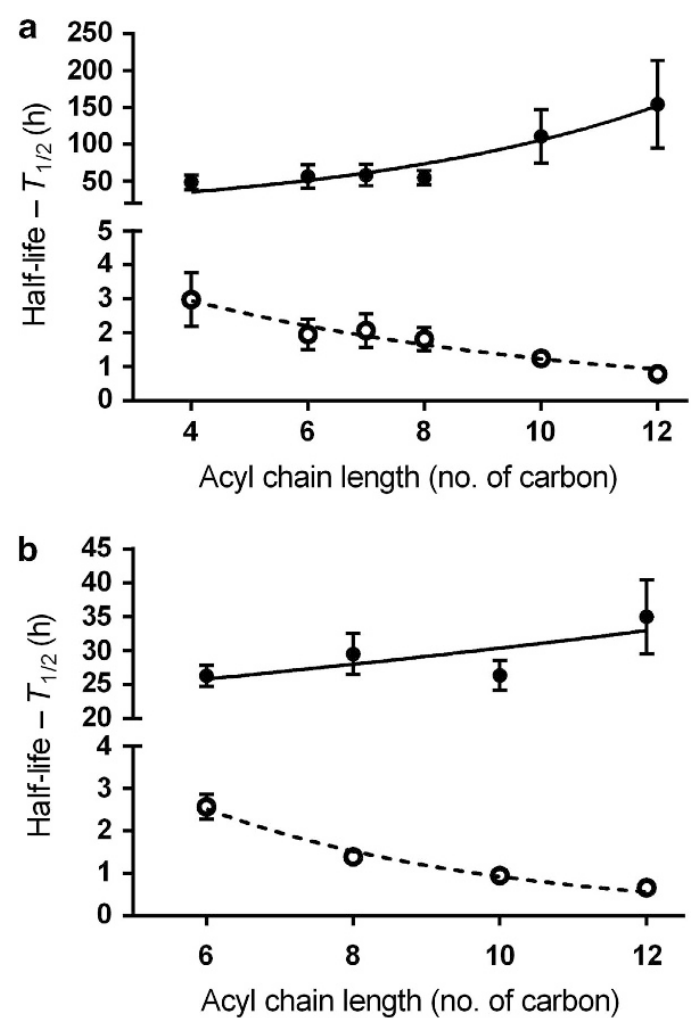

Figure 1. Degradation of $\mathrm{N}$-acyl homoserine lactones (AHLs) based on the function of acyl chain length in the floccular sludge. The degradation value is expressed as the signal half-life. A mixture of unsubstituted (a) and oxo-substituted (b) AHLs was added to the live (open circle) or the heat-inactivated (solid circle) sludge to a final concentration of $5 \mu \mathrm{M}$ for each $\mathrm{AHL}$. The sludge mixture was incubated at room temperature with constant shaking at 200 r.p.m. The $\mathrm{pH}$ of each sludge mixture (bulk liquid) was monitored throughout the study and ranged between $\mathrm{pH} 6.7$ and 6.9. Residual AHLs were extracted and quantified using LC-MS/MS at different time points. The signal half-life for each AHL was estimated based on the zero-order or first-order kinetics. Error bars are defined as s.e.m. ( $n=3$, biological replicates). Holm-Sidak multiple $t$-tests were performed (live versus heat-inactivated control for each $\mathrm{AHL}$ ) and corrected $P$ values ( $P<0.05$ for all pairs of comparison) are reported.

producer was subsequently determined using LC-MS/MS. AHL degradation was assayed using AHLs with short (3OC6-HSL), medium (3OC8-HSL) and long (3OC12-HSL) acyl chains. A large proportion of floccular sludge isolates, $65 \%$, were either AHL signal producers or quenchers (Figure 2). Although only $10 \%$ of the isolates could produce AHLs, as many as $58.1 \%$ of the isolates were able to quench at least one of the AHLs examined. A relatively small proportion of the isolates, $4.8 \%$, both produced and quenched AHLs. The remaining $36.7 \%$ of isolates neither produced nor quenched AHLs. All of the $\mathrm{AHL}$ quenchers were capable of inactivating the long-chain AHL, 3OC12-HSL. Although $77 \%$ of the quenchers could only degrade the long-chain AHLs, $\sim 23 \%$ could degrade long-chain AHLs as well as short or medium-chain AHLs.

AHL quenching community members are phylogenetically diverse and distinct from AHL producers

The evolutionary relationships among the bacterial isolates, based on their $16 \mathrm{~S}$ rDNA sequences, indicated that there was no specific clade associated with $\mathrm{QQ}$ activity, while the $\mathrm{AHL}$ producers were all classified as alpha, beta or gamma Proteobacteria (Figure 3).

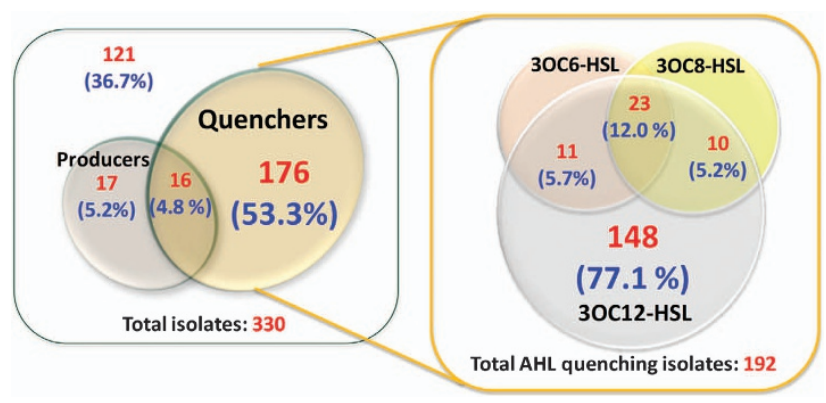

Figure 2. Distribution of isolates according to their capacity to produce and/or quench $\mathrm{N}$-acyl homoserine lactones (AHLs). A total of 330 isolates, including 307 and 23 strains of bacterial and fungal origins, respectively, were cultured from the floccular sludge community during the bioreactor operation from weeks 40-44. All isolates were screened for $\mathrm{AHL}$ production by different bioassays and LC-MS/MS, as well as for their capability to quench $5 \mu \mathrm{M}$ of AHLs with short (3OC6-HSL), medium (3OC8-HSL) and long (3OC12-HSL) acyl chains after $2 \mathrm{~h}$ incubation. Residual AHLs were quantified using the A. tumefaciens A136 agar-based spot bioassay.

These QS isolates represented genera that have previously been reported to be AHL producers, such as Acidovorax, Pantoea, Rhizobium, Rhodobacter, Sphingomonas and Stenotrophomonas, as well as novel genera that have not been reported to synthesise AHLs, including Frateuria, Lysobacter and Shinella (Figure 3). The LC-MS/MS analysis further revealed that the isolates produced AHLs of various acyl chain lengths ranging from 4 to 14 carbons (Supplementary Table S5). Every signal producing isolate synthesised more than one type of AHL and multiple genera/species could produce the same AHL (Supplementary Tables S2 and 5). For example, isolates from six out of nine bacterial genera were capable of synthesising 30C8-HSL (Supplementary Table S5), the dominant signal detected in the sludge community in situ (Supplementary Figure S4). In contrast, the AHL quenchers were classified into four different bacterial phyla, including the Proteobacteria, Actinobacteria, Bacteriodetes and Firmicutes, as well representatives from the Fungi (Figure 3 and Supplementary Table S2). Although some of the $\mathrm{QQ}$ isolates represented genera that are commonly reported to degrade AHLs, such as Ochrobacterium, Variovorax, Bacillus and Rhodoccus, most of the $\mathrm{QQ}$ isolates identified here have not previously been shown to have this activity. These novel $\mathrm{QQ}$ organisms were distributed throughout the genera Novosphingobium, Acidovorax, Rheinheimera, Tsukamurella, Flavobacterium and Candida, among others. Interestingly, some of the isolates were capable of both synthesising and degrading AHLs, e.g., Rhizobium borbori N065. In addition, there was considerable variation in function even at the species level. For example, 10 strains of Stenotrophomonas sp. with identical $16 \mathrm{~S}$ rDNA sequences were able to produce AHLs, while the remaining six strains were not (Supplementary Table S2). Likewise, not every strain of the same species was able to quench AHLs, as was the case for Stenotrophomonas maltophilia. The specific QS and QQ characteristics of each isolate implies that AHL signalling at the community level is complex, and varying QS and QQ species composition may have different impacts on various signalling-regulated community behaviours.

Community shift from signal quenchers to producers may lead to signal accumulation and granular biofilm assembly

To explore the role of species composition and signalling on community function, in terms of biofilm structure, the signalling properties of two independent communities, i.e., the floccular and the granular sludge communities ${ }^{19}$ were compared. Both floccular and granular sludge bioreactors were first seeded with the simultaneous nitrification, denitrification and phosphorus 


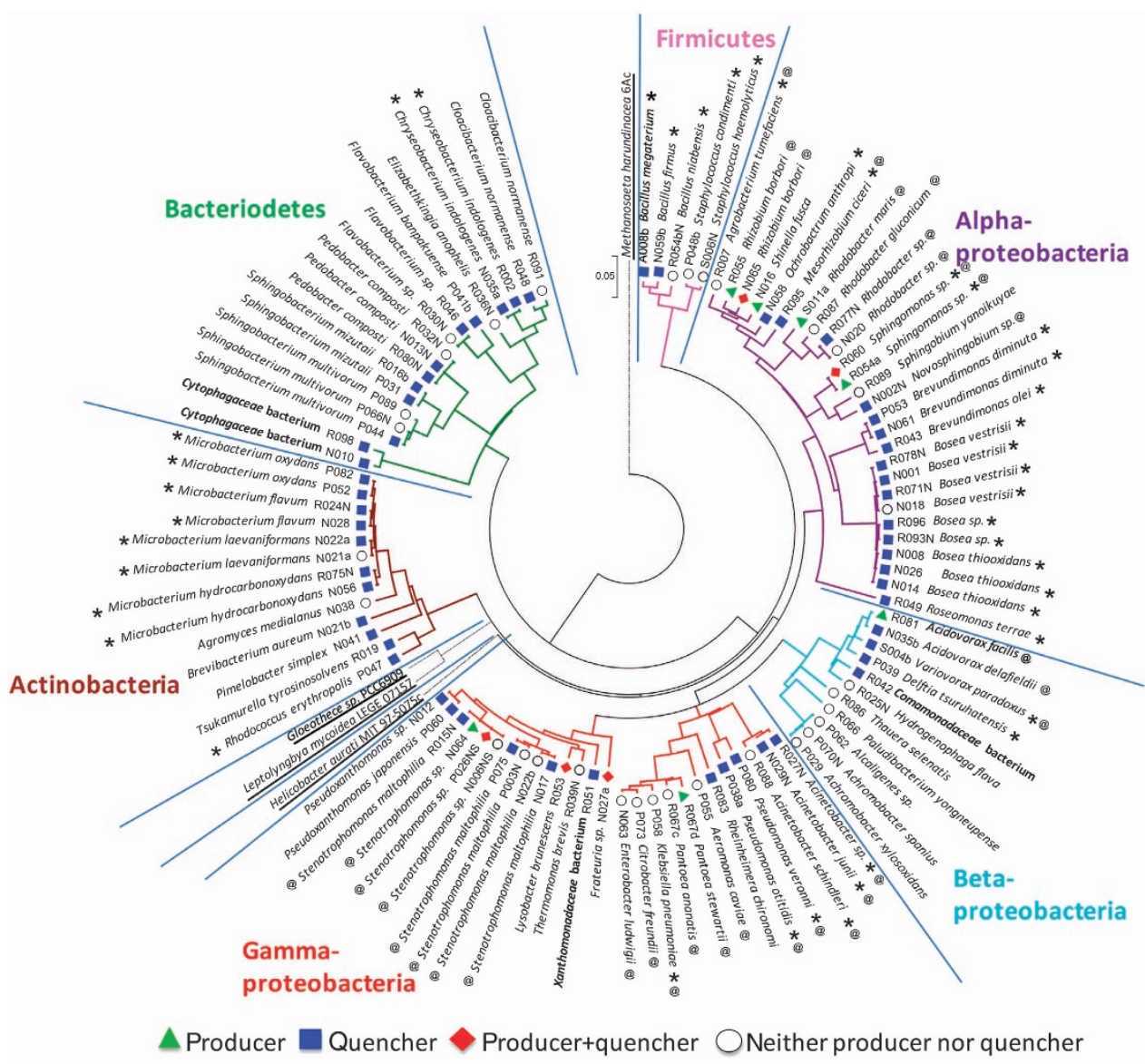

Figure 3. Evolutionary relationship of 100 representative bacterial isolates (taxa) based on the $16 \mathrm{~S}$ rDNA gene sequences. Each representative was selected for their uniqueness in terms of $\mathrm{N}$-acyl homoserine lactone (AHL) production and/or quenching properties. The evolutionary relationship was inferred using the neighbour-joining method. The bootstrap consensus tree inferred from 1,000 replicates was taken to represent the evolutionary relationship of the taxa analysed. Branches corresponding to partitions reproduced in $<50 \%$ bootstrap replicates were collapsed. The tree was drawn to scale, with branch lengths reflecting evolutionary distances. The evolutionary distances were computed using the maximum composite likelihood method and are in the units of the number of base substitutions per site. All positions containing gaps and missing data were eliminated from the data set (complete deletion option). There were a total of 1,031 positions in the final data set. Phylogenetic analyses were conducted in MEGA4. The outgroups, retrieved from the GenBank database, are underlined. The isolated strains are classified as producer (green triangle), quencher (blue square), producer and quencher (red diamond) and neither producer nor quencher (open circle). The genera in which AHL production (@) or AHL degradation $(*)$ activities have been reported in the literature are indicated. The closest relative with at least $97 \%$ sequence identity for each strain, except for those strains highlighted in bold, is shown.

removal floccular sludge inoculum, ${ }^{24}$ and were either operated to maintain the floccular sludge or the conditions were changed to facilitate the formation of microbial granules. ${ }^{19}$ The species composition and the signal profile of both communities were characterised over time. Pearson correlation analysis of the top 50 most abundant floccular sludge community members (including non-cultivable members by RNA-sequencing) and the concentration of individual AHLs in situ revealed the dominance of community members that were negatively correlated with $\mathrm{AHL}$ production over 82 weeks (Figure 4, left panel, Cluster 3). Approximately $72 \%$ of the Pearson relationships were classified as negative, whereas only $20 \%$ were positive at the floccular stage (Figure 4, left panel). The high percentage of negative relationships was consistent with the higher proportions of $\mathrm{QQ}$ isolates in the floccular sludge community (Figures 2 and 3). When flocs were transformed into granules, there was a significant decrease in the negative correlation between community members and $\mathrm{AHL}$ concentration, from 72 to $38 \%$, concomitant with an increase from 20 to $54 \%$ of positive relationships (Figure 4, right panel). ${ }^{19}$ These findings thus indicate a distinct community shift from potential signal quenchers to producers during the transition from flocs to granules. Accordingly, the $\mathrm{QQ}$ activity of the granular sludge was substantially reduced relative to the floccular sludge (Supplementary Figure S5). For example, the half-life of 30C8HSL was $\sim 28 \%$ longer for the granular sludge compared with the floccular sludge. In addition, AHLs, including 3OC6-HSL, 3OC8-HSL, C6-HSL and C8-HSL, accumulated to at least a threefold higher concentration during the granular stage compared with the floccular stage ( $P<0.05$ for all AHLs) (Supplementary Figure S6). In particular, the dominant signal 30C8-HSL increased from an average of $25 \mathrm{pmol} / \mathrm{g}$ at the floccular stage to $85 \mathrm{pmol} / \mathrm{g}$ at the granular stage $(P<0.016)$. The elevated amount of AHLs was previously associated with the increased expression of extracellular polymeric substances and the formation of microbial granules, whereas decreased $\mathrm{AHL}$ levels were linked to granular dispersal and conversion to floccular biomass. ${ }^{19}$ Here it appears that signal gradients could be regulated by the QQ activity of the community depending on the species composition and the associated QQ capacity. It is therefore possible that the balance between the competing $\mathrm{QS}$ and $\mathrm{QQ}$ activities may determine the community signalling behaviour or function, e.g., the assembly of microbial granular biofilms. ${ }^{19}$ 


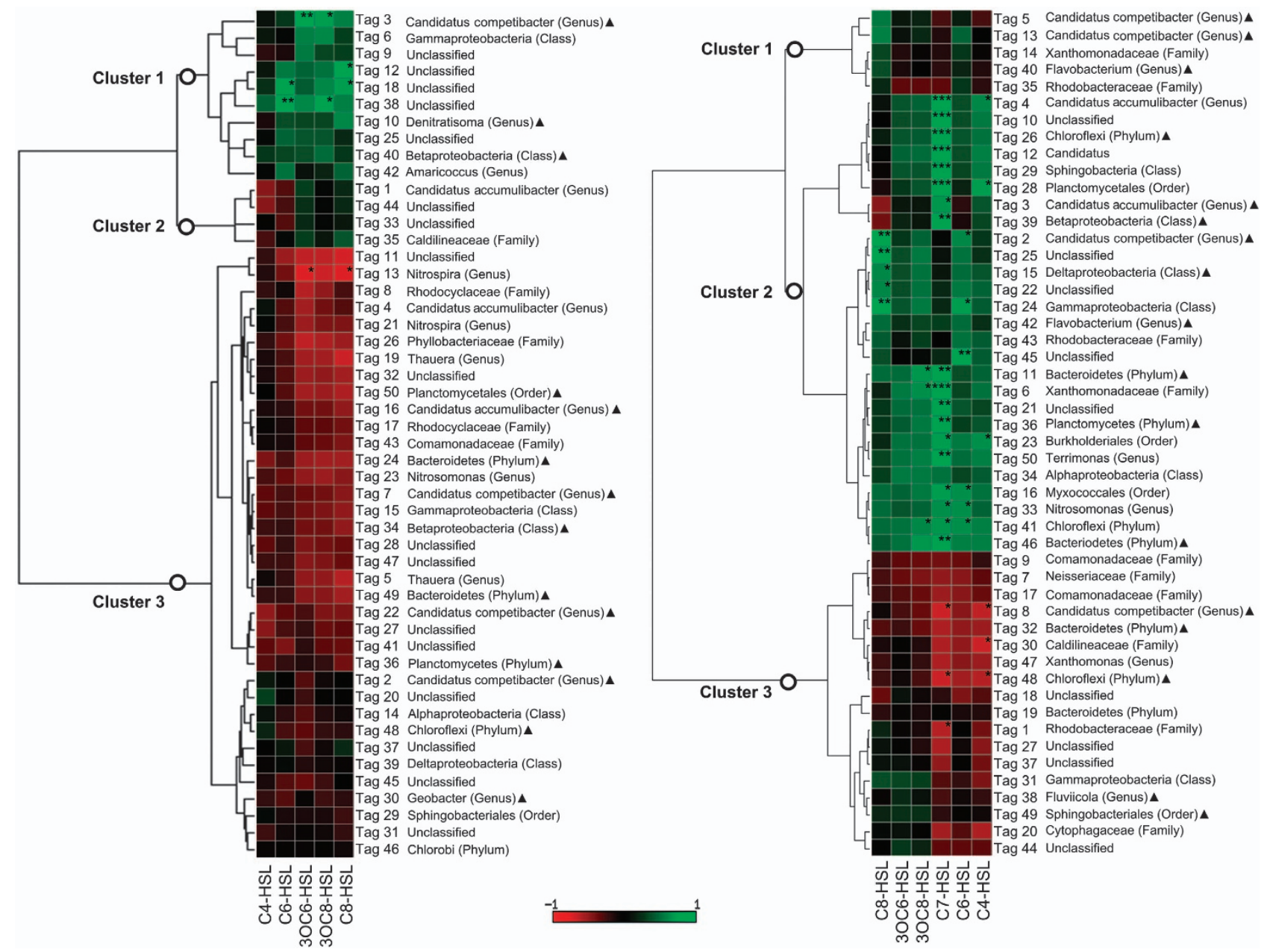

Figure 4. A comparison between the signalling properties of the floccular (left panel) and the granular (right panel) sludge communities. The relationships between the abundance of the top 50 most dominant community members (each represented by a unique V6 tag) and the concentration profile of $\mathrm{N}$-acyl homoserine lactones (AHLs) over time were calculated based on Pearson correlation for the respective floccular and granular sludge communities. The Pearson correlation coefficient is colour coded, indicating relationships from strongly positive

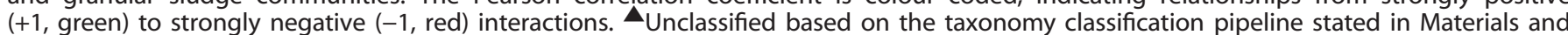
methods section. The closest relative with sequence identity matching $>97 \%$ is shown where it is applicable. False discovery rate corrections for multiple comparisons were performed, and significant differences are indicated as follows: ${ }^{*} P<0.05,{ }^{* *} P<0.01$ and ${ }^{* * *} P<0.001$. The signalling properties of the granular sludge community was adapted from Tan et al. ${ }^{19}$

\section{DISCUSSION}

The study of interspecies interactions is important for developing a systematic understanding of the physiology of high-density, matrix-encased microbial communities, which are now widely appreciated to be the dominant mode of growth for bacteria in natural and engineered ecosystems., ${ }^{3,42}$ Communication via diffusible signalling molecules has been proposed to be one of the important interactions among species of diverse origins, ${ }^{3}$ and there is growing evidence that complex communities in natural environments produce QS signals in situ to regulate different community behaviours. ${ }^{14,16,18,19}$ Although these findings have demonstrated the roles of QS in complex communities, the question of how competing activities, such as the $\mathrm{QQ}$ modulation of QS in complex communities, are mediated remains to be addressed. Using microbial granular assembly as a model for QS-mediated community behaviour, ${ }^{19}$ this study has shown that QQ strongly suppresses QS signal accumulation in the floccular sludge (Figure 1), and may thus inhibit the formation of microbial granules. When the QQ suppression was partially relieved, indicated by the increased signal half-lives at the granular stage (Supplementary Figure S5), the signal concentrations were increased significantly (Supplementary Figure S6), suggesting that
QQ can be an effective modulator of community QS signalling. Indeed, addition of exogenous QQ enzymes to a membrane bioreactor can delay mixed-species biofilm development on membrane surfaces. ${ }^{18,43}$ In our study, we further established that QQ is an inherent function of complex communities that may have a strong influence on many interspecies interactions based on $\mathrm{AHL}$ signalling. Therefore, in contrast to some natural habitats where environmental factors have a dominant role in regulating the levels of QS signals, such as photosynthesis-driven cycles of high $\mathrm{pH}$ (up to 9.4), which strongly attenuates AHL signalling in microbial mats, ${ }^{14}$ we found that $\mathrm{pH}$ (up to $<8.3$ ) and physical signal adsorption were less important than enzymatic activity in the inactivation of signals in our model system (Supplementary Figures S2 and 3). This therefore suggests that QQ activity was the primary mechanism of signal removal, and hence serves as a key regulator of signal levels at different stages of granulation life cycle.

Based on the results obtained in this study, we submit that the overall $\mathrm{AHL}$ accumulation in the granulation system was directly related to the relative proportions and the activities of QQ and QS organisms in the community. As many as $60 \%$ of isolates from the floccular sludge community, representing 32 bacterial and 4 
fungal genera were signal quenchers, while only $10 \%$ of the isolates (9 bacterial genera) produced AHLs (Figures 2 and 3). Whereas the proportion of isolated AHL producers observed here is consistent with observations from other environments, ${ }^{7,8}$ the frequency of $\mathrm{AHL}$ quenchers is considerably higher than reported in other studies ( $\sim 5-15 \%$ of isolates). ${ }^{7,8}$ The higher proportion of $\mathrm{AHL}$ quenchers detected here is likely a consequence of testing for $\mathrm{QQ}$ activity using a range of AHLs as targets, including the longchain AHLs, which have not typically been used to detect QQ activity. Interestingly, AHLs were degraded in a chain lengthdependent manner in situ where the long-chain AHLs were preferentially inactivated (Figure 1), which is consistent with the observations of a higher proportion of isolates being able to degrade long-chain AHLs (100\% of AHL quenchers) as compared with short- and medium-chain AHLs ( 23\% of AHL quenchers; Figure 2). Thus, the QQ activities of the spectrum of isolates collected were more generally reflected in the types of signals that were degraded by the whole community in situ. This observation also corresponded with the profile of AHLs that were identified in the floccular sludge community, where only AHLs with short or medium acyl chains were detected at low concentrations, while signals with long acyl chains $\geqslant 10$ carbons were not detected at all (Supplementary Figure S4). Almost every QS isolate produced at least one long-chain AHL (Supplementary Table S5), suggesting that the sludge community has the capacity to synthesise the long-chain signals in situ. Thus, the lack of long-chain AHLs in the sludge was not due to the absence of producer strains, and is most likely a consequence of specific QQ activity by the community.

The isolation studies indicated that the floccular sludge community was dominated by the signal quenchers $160 \%$; Figure 2), and this was also reflected in the whole-community analysis where $>70 \%$ of the top 50 most abundant community members were negatively correlated with $\mathrm{AHL}$ accumulation (Figure 4; left panel-Cluster 3). Although many factors may have contributed to the negative relationships, one key factor could be that Cluster 3 community members were potential quenchers that antagonise signal accumulation. In support of this, one of the cluster members, Tag 43 (Diaphorobacter nitroreducens R042), was isolated and confirmed to be an AHL quencher (Supplementary Table S2). The higher proportions of signal quenchers relative to the producers in the whole community may thus explain the short half-lives of AHLs, and hence the low AHL concentrations at the floccular stage (Figure 1 and Supplementary Figure S6). Conversely, there was a shift in the community towards QS, i.e., positive correlations, with a concomitant decrease in $\mathrm{QQ}$ organisms, i.e., negative correlations, when the biomass was transformed from flocs to granules (Figure 4, right panel). For example, Lysobacter brunescens R037 (Figure 4, right panel-Tag 6 in granular sludge), a signal producer that has been isolated from the community, was almost undetectable at the floccular stage but was highly abundant during granulation. ${ }^{19}$ In contrast, the signal quencher, D. nitroreducens R042 (Figure 4; Tag 43 in the floccular sludge, which is equivalent to Tag 17 in the granular sludge) was highly abundant in the floccular sludge and was decreased substantially during granulation. ${ }^{19}$ Although it is not yet possible to determine whether the community shift from the potential QQ organisms to QS organisms is a direct cause of granulation, or whether granulation favours the enrichment of QS organisms over the QQ species, the strong associations between both QS-dominated communities and granules, and QQ-dominated communities and flocs, suggest that differential $\mathrm{AHL}$ signalling is important in community level functions, such as granulation.

The co-occurrence of $\mathrm{QS}$ and $\mathrm{QQ}$ activities identified here is likely to be common across many habitats. Indeed, organisms known to be $\mathrm{AHL}$ producers or quenchers, including Proteobacteria, Bacteriodetes, Firmicutes and Actinobacteria phyla, have been recently expanded to also include Cyanobacteria, ${ }^{44,45}$ Acidobacteria, ${ }^{46}$ Bacteriodetes ${ }^{47-49}$ and Archaea ${ }^{50}$ from a diverse range of environments. Further, many of these species co-exist in the same niches, such as the rumen, ${ }^{15,51}$ microbial mats ${ }^{14}$ and the soil/rhizosphere. ${ }^{7,8}$ This is also likely the case for used water treatment facilities, which harbour very high microbial diversity, including many QS and QQ organisms as identified in this study and in the literature. ${ }^{6,17}$ Indeed, our preliminary metagenomic analysis and expression studies suggest that multiple QS and QQ genes are present in the activated sludge community from a full-scale plant (data not shown), not only supporting the cooccurrence of QS and QQ activities in the bioreactor community but also that community behaviour is controlled by the combined activity of the QS and QQ organisms present, as well as the specificity of the $\mathrm{QQ}$ activity for particular AHL signals.

In conclusion, it is clear from this study that while a positive correlation between $\mathrm{AHL}$ signalling and the assembly of microbial granular biofilms has been previously established, ${ }^{19}$ the detailed mechanisms by which community members with distinct QS and QQ activities interact to regulate QS behaviours at the community level remain to be defined. This study has developed the granulation system as an experimentally tractable system comprising high species diversity to investigate and determine, in a mechanistic fashion, how such communities control complex system level behaviours, such as granulation. Our study provides strong evidence that AHL-mediated QS signalling is a true community trait, with $>65 \%$ of community members of diverse taxa engaged in the signalling and quenching processes. The dynamic abundance of QS and QQ organisms, as well as their respective signalling and quenching kinetics, appear to have a key role for defining the overall signal gradient, and hence regulate the transition from floccular to granular biofilms. Importantly, these findings may also imply that complex community assembly is a developmental process driven by QS signalling, with the enrichment of specific community members with QS signalling properties and exclusion of members with anti-QS signalling traits. The observation that QS and QQ activities are associated with phylogenetically diverse microbial species that are commonly found in many different natural habitats, in engineered ecosystems as well as in clinical settings, strongly suggests that AHL-mediated community communication could be a general feature of complex communities, and that the QS and QQ activities may have a significant role in the selection of stable and functional microbial assemblages.

\section{ACKNOWLEDGEMENTS}

We thank Yehuda Cohen for providing valuable feedback on the manuscript. Special acknowledgement to Zhaoqi Zhan, Peiting Zeng and Edwin Ting for their help with AHL quantification, and Shimazdu Singapore for providing the use of LC-MS/MS. CHT was supported by National Research Foundation (NRF) Singapore under its NRF Environmental and Water Technologies PhD Scholarship Programme and administered by the Environment and Water Industry Programme Office. The research was supported by the Singapore Centre for Environmental Life Sciences Engineering, whose research is funded by the NRF and Ministry of Education of Singapore, Nanyang Technological University and National University of Singapore, under its Research Centre of Excellence Programme.

\section{CONTRIBUTIONS}

CHT, KSK, YZ, WJN, SAR and SK designed the research; CHT, JZ, XHT and GPL performed the research; CHT, KSK, CX, YZ, WJN, SAR and SK analysed the data; and $\mathrm{CHT}$, SAR and SK wrote the paper.

\section{COMPETING INTERESTS}

The authors declare no conflict of interest. 


\section{REFERENCES}

1 Davey ME, O'toole GA. Microbial biofilms: from ecology to molecular genetics. Microbiol Mol Biol Rev 2000; 64: 847-867.

2 Kolter R, Greenberg EP. Microbial sciences: the superficial life of microbes. Nature 2006; 441: 300-302.

3 Elias S, Banin E. Multi-species biofilms: living with friendly neighbors. FEMS Microbiol Rev 2012; 36: 990-1004.

4 Shapiro JA. Thinking about bacterial populations as multicellular organisms. Annu Rev Microbiol 1998; 52: 81-104.

5 Williams P, Winzer K, Chan WC, Cámara M. Look who's talking: communication and quorum sensing in the bacterial world. Philos Trans R Soc Lond B Biol Sci 2007; 362: 1119-1134.

6 Valle A, Bailey MJ, Whiteley AS, Manefield M. N-acyl-I-homoserine lactones (AHLs) affect microbial community composition and function in activated sludge. Environ Microbiol 2004; 6: 424-433.

7 d'Angelo-Picard C, Faure D, Penot I, Dessaux Y. Diversity of $\mathrm{N}$-acyl homoserine lactone-producing and -degrading bacteria in soil and tobacco rhizosphere. Environ Microbiol 2005; 7: 1796-1808.

8 Cirou A, Diallo S, Kurt C, Latour X, Faure D. Growth promotion of quorumquenching bacteria in the rhizosphere of Solanum tuberosum. Environ Microbiol 2007; 9: 1511-1522.

9 Pierson EA, Wood DW, Cannon JA, Blachere FM, Pierson LS. Interpopulation signaling via $\mathrm{N}$-acyl-homoserine lactones among bacteria in the wheat rhizosphere. Mol Plant Microbe Interact 1998; 11: 1078-1084.

10 Riedel K, Hentzer M, Geisenberger O, Huber B, Steidle A, Wu H et al. $\mathrm{N}$-acylhomoserine-lactone-mediated communication between Pseudomonas aeruginosa and Burkholderia cepacia in mixed biofilms. Microbiology 2001; 147: 3249-3262.

11 Dulla GF, Lindow SE. Acyl-homoserine lactone-mediated cross talk among epiphytic bacteria modulates behavior of Pseudomonas syringae on leaves. ISME J 2009; 3: 825-834.

12 Hosni T, Moretti C, Devescovi G, Suarez-Moreno ZR, Fatmi MB, Guarnaccia C et al. Sharing of quorum-sensing signals and role of interspecies communities in a bacterial plant disease. ISME J 2011; 5: 1857-1870.

13 Singh PK, Schaefer AL, Parsek MR, Moninger TO, Welsh MJ, Greenberg EP et al. Quorum-sensing signals indicate that cystic fibrosis lungs are infected with bacterial biofilms. Nature 2000; 407: 762-764.

14 Decho AW, Visscher PT, Ferry J, Kawaguchi T, He L, Przekop KM et al. Autoinducers extracted from microbial mats reveal a surprising diversity of $\mathrm{N}$-acylhomoserine lactones (AHLs) and abundance changes that may relate to diel $\mathrm{pH}$. Environ Microbiol 2009; 11: 409-420.

15 Hughes DT, Terekhova DA, Liou L, Hovde CJ, Sahl JW, Patankar AV et al. Chemical sensing in mammalian host-bacterial commensal associations. Proc Natl Acad Sci USA 2010; 107: 9831-9836.

16 De Clippeleir H, Defoirdt T, Vanhaecke L, Vlaeminck SE, Carballa M, Verstraete W et al. Long-chain acylhomoserine lactones increase the anoxic ammonium oxidation rate in an OLAND biofilm. Appl Microbiol Biotechnol 2011; 90: 1511-1519.

17 Chong G, Kimyon O, Rice SA, Kjelleberg S, Manefield M. The presence and role of bacterial quorum sensing in activated sludge. Microbial Biotechnol 2012; 5: 621-633.

18 Yeon KM, Cheong WS, Oh HS, Lee WN, Hwang BK, Lee CH et al. Quorum sensing: a new biofouling control paradigm in a membrane bioreactor for advanced wastewater treatment. Environ Sci Technol 2009; 43: 380-385.

19 Tan CH, Koh KS, Xie C, Tay M, Zhou Y, Williams R et al. The role of quorum sensing signalling in EPS production and the assembly of a sludge community into aerobic granules. ISME J 2014; 8: 1186-1197.

20 Dong YH, Wang LH, Xu JL, Zhang HB, Zhang XF, Zhang LH et al. Quenching quorum-sensing-dependent bacterial infection by an $\mathrm{N}$-acyl homoserine lactonase. Nature 2001; 411: 813-817.

21 Lin $\mathrm{YH}, \mathrm{Xu} J \mathrm{~L}, \mathrm{Hu}$ J, Wang LH, Ong SL, Leadbetter JR et al. Acyl-homoserine lactone acylase from Ralstonia strain $\mathrm{XJ12B}$ represents a novel and potent class of quorum-quenching enzymes. Mol Microbiol 2003; 47: 849-860.

22 Park SY, Kang HO, Jang HS, Lee JK, Koo BT, Yum DY et al. Identification of extracellular $\mathrm{N}$-acylhomoserine lactone acylase from a Streptomyces $\mathrm{sp}$ and its application to quorum quenching. Appl Environ Microbiol 2005; 71: 2632-2641.

23 Uroz S, Oger PM, Chapelle E, Adeline MT, Faure D, Dessaux YA. Rhodococcus qsdAencoded enzyme defines a novel class of large-spectrum quorum-quenching lactonases. Appl Environ Microbiol 2008; 74: 1357-1366.

24 Zhou Y, Ganda L, Lim M, Yuan Z, Kjelleberg S, Ng WJ et al. Free nitrous acid (FNA) inhibition on denitrifying poly-phosphate accumulating organisms (DPAOs). Appl Microbiol Biotechnol 2010; 88: 359-369.
25 Smolders GJ, van der Meij J, van Loosdrecht MC, Heijnen JJ. Model of the anaerobic metabolism of the biological phosphorus removal process: stoichiometry and pH influence. Biotechnol Bioeng 1994; 43: 461-470.

26 Eaton AD, Franson MAH, Association APH, Association AWW, Federation WE. Standard Methods for the Examination of Water and Wastewater. American Public Health Association: Washington D.C., USA, 2005.

27 Barr JJ, Cook AE, Bond PL. Granule formation mechanisms within an aerobic wastewater system for phosphorus removal. Appl Environ Microbiol 2010; 76: 7588-7597.

28 Fuqua C, Burbea M, Winans SC. Activity of the Agrobacterium Ti plasmid conjugal transfer regulator TraR is inhibited by the product of the traM gene. $J$ Bacteriol 1995; 177: 1367-1373.

29 McClean KH, Winson MK, Fish L, Taylor A, Chhabra SR, Camara M et al. Quorum sensing and Chromobacterium violaceum: exploitation of violacein production and inhibition for the detection of $\mathrm{N}$-acylhomoserine lactones. Microbiology 1997; 143 3703-3711.

30 Andersen JB, Heydorn A, Hentzer M, Eberl L, Geisenberger O, Christensen BB et al. gfp-based $\mathrm{N}$-acyl homoserine-lactone sensor systems for detection of bacterial communication. Appl Environ Microbiol 2001; 67: 575-585.

31 Heydorn A, Ersbøll B, Kato J, Hentzer M, Parsek MR, Tolker-Nielsen T et al. Statistical analysis of Pseudomonas aeruginosa biofilm development: impact of mutations in genes involved in twitching motility, cell-to-cell signaling, and stationary-phase sigma factor expression. Appl Environ Microbiol 2002; 68: 2008-2017.

32 Yanisch-Perron C, Vieira J, Messing J. Improved M13 phage cloning vectors and host strains: nucleotide sequences of the M13mp18 and pUC19 vectors. Gene 1985; 33: 103-119.

33 Wang LH, Weng LX, Dong YH, Zhang LH. Specificity and enzyme kinetics of the quorum-quenching $\mathrm{N}$-Acyl homoserine lactone lactonase (AHL-lactonase). J Biol Chem 2004; 279: 13645-13651.

34 Morin D, Grasland B, Vallée-Réhel K, Dufau C, Haras D. On-line high-performance liquid chromatography-mass spectrometric detection and quantification of $\mathrm{N}$-acylhomoserine lactones, quorum sensing signal molecules, in the presence of biological matrices. J Chromatogr A 2003; 1002: 79-92.

35 Clark JD, Maaloe O. DNA replication and the division cycle in Escherichia coli. J Mol Biol 1967; 23: 99-112.

36 Lane DJ. 16S/23S rRNA Sequencing. John Wiley \& Sons: New York, USA, 1991.

37 Gardes M, White TJ, Fortin JA, Bruns TD, Taylor JW. Identification of indigenous and introduced symbiotic fungi in ectomycorrhizae by amplification of nuclear and mitochondrial ribosomal DNA. Can J Bot 1991; 69: 180-190.

38 Dong $\mathrm{YH}, \mathrm{Xu} \mathrm{JL}, \mathrm{Li} \mathrm{XZ}$, Zhang $\mathrm{LH}$. AiiA, an enzyme that inactivates the acylhomoserine lactone quorum-sensing signal and attenuates the virulence of Erwinia carotovora. Proc Natl Acad Sci USA 2000; 97: 3526-3531.

39 Zhang HB, Wang LH, Zhang LH. Detection and Analysis of Quorum-Quenching Enzymes Against Acyl Homoserine Lactone Quorum-Sensing Signals. John Wiley and Sons, Inc: Hoboken, New Jersey, USA, 2007.

40 Saitou N, Nei M. The neighbor-joining method: a new method for reconstructing phylogenetic trees. Mol Biol Evol 1987; 4: 406-425.

41 Claesson MJ, Wang Q, O'Sullivan O, Greene-Diniz R, Cole JR, Ross RP et al. Comparison of two next-generation sequencing technologies for resolving highly complex microbiota composition using tandem variable $16 \mathrm{~S}$ rRNA gene regions. Nucleic Acids Res 2010; 38: e200.

42 Straight PD, Kolter R. Interspecies chemical communication in bacterial development. Annu Rev Microbiol 2009; 63: 99-118.

43 Oh H-S, Yeon K-M, Yang C-S, Kim S-R, Lee C-H, Park SY et al. Control of membrane biofouling in MBR for wastewater treatment by quorum quenching bacteria encapsulated in microporous membrane. Environ Sci Technol 2012; 46: 4877-4884.

44 Romero M, Diggle SP, Heeb S, Cámara M, Otero A. Quorum quenching activity in Anabaena sp. PCC 7120: identification of AiiC, a novel AHL-acylase. FEMS Microbiol Lett 2008; 280: 73-80.

45 Sharif DI, Gallon J, Smith CJ, Dudley E. Quorum sensing in Cyanobacteria: $\mathrm{N}$-octanoyl-homoserine lactone release and response, by the epilithic colonial cyanobacterium Gloeothece PCC6909. ISME J 2008; 2: 1171-1182.

46 Bijtenhoorn P, Mayerhofer H, Müller-Dieckmann J, Utpatel C, Schipper C, Hornung $\mathrm{C}$ et al. A novel metagenomic short-chain dehydrogenase/reductase attenuates Pseudomonas aeruginosa biofilm formation and virulence on Caenorhabditis elegans. PLoS One 2011; 6: e26278.

47 Huang YL, Ki JS, Case RJ, Qian PY. Diversity and acyl-homoserine lactone production among subtidal biofilm-forming bacteria. Aquat Microb Ecol 2008; 52: 185-193.

48 Romero $M$, Avendaño-Herrera $R$, Magariños $B$, Cámara $M$, Otero $A$. Acylhomoserine lactone production and degradation by the fish pathogen Tenacibaculum maritimum, a member of the Cytophaga-FlavobacteriumBacteroides (CFB) group. FEMS Microbiol Lett 2010; 304: 131-139. 
49 Rashid R, Morohoshi T, Someya N, Ikeda T. Degradation of $\mathrm{N}$-acylhomoserine lactone quorum sensing signaling molecules by potato root surface-associated Chryseobacterium strains. Microbes Environ 2011; 26: 144-148.

50 Zhang G, Zhang F, Ding G, Li J, Guo X, Zhu J et al. Acyl homoserine lactone-based quorum sensing in a methanogenic archaeon. ISME J 2012; 6: 1336-1344.

51 Koenig JE, Spor A, Scalfone N, Fricker AD, Stombaugh J, Knight R et al. Succession of microbial consortia in the developing infant gut microbiome. Proc Natl Acad Sci USA 2011; 108: 4578-4585. (c) (i) This work is licensed under a Creative Commons Attribution 4.0 International License. The images or other third party material in this article are included in the article's Creative Commons license, unless indicated otherwise in the credit line; if the material is not included under the Creative Commons license, users will need to obtain permission from the license holder to reproduce the material. To view a copy of this license, visit http://creativecommons.org/licenses/ by/4.0/

Supplementary Information accompanies the paper on the npj Biofilms and Microbiomes website (http://www.nature.com/npjbiofilms) 